Original Paper http://ajol.info/index.php/ijbcs http://indexmedicus.afro.who.int

\title{
Validation de la méthode de détermination d'aflatoxine B1 dans les pâtes d'arachide vendues sur les marchés de la ville d'Abidjan (Côte d'Ivoire)
}

\author{
Zamblé Bi Irié Abel BOLI ${ }^{1 *}$, Fatoumata CAMARA ${ }^{2}$, Djegba Marie TOKA ${ }^{1}$, \\ Marina KOUSSEMON $^{2}$ et Rose KOFFI-NEVRY ${ }^{1}$ \\ ${ }^{1}$ Laboratoire de Biotechnologie et Microbiologie Alimentaire, UFR des Sciences et Technologie des \\ Aliments, Université Nangui Abrogoua, 02 BP 801, Abidjan 02, Côte d'Ivoire. \\ ${ }^{2}$ Laboratoire de Nutrition et Sécurité Alimentaire, UFR des Sciences et Technologie des Aliments, \\ Université Nangui Abrogoua, 02 BP 801, Abidjan 02, Côte d'Ivoire. \\ *Auteur correspondant, E-mail: bzia2011@yahoo.fr
}

\section{RESUME}

Cette étude a été conduite pour déterminer les paramètres de validation de la méthode de dosage d'Aflatoxine B1 dans les pâtes d'arachide par chromatographie liquide haute performance (HPLC) selon la norme NF V03-110 Mai 2010. Les tests de la validation ont été réalisés à partir de dix échantillons de pâte d'arachide. La linéarité de la courbe d'étalonnage établie entre 0 et 3,0 $\mu \mathrm{g} / \mathrm{L}$ a été prouvée en déterminant le carré du coefficient de corrélation de Pearson $\left(\mathrm{R}^{2}=0,9985\right)$. Les limites de détection et de quantification sont de $0,01 \mu \mathrm{g} / \mathrm{kg}$ et $0,02 \mu \mathrm{g} / \mathrm{kg}$ respectivement. Les coefficients de variation des tests de répétabilité et de reproductibilité sont respectivement de $3,4 \%$ et $3,79 \%$. Le taux de récupération est estimé à $107,6 \%$ et se situe dans la limite acceptable à l'échelle internationale (70-110\%). Le test de conformité n'a pas montré de différence significative $(\mathrm{P}>0,05)$ entre la concentration moyenne en aflatoxine $\mathrm{B} 1$ obtenues dans les échantillons de référence de pâte d'arachide $(2,02 \pm 1,44 \mu \mathrm{g} / \mathrm{kg})$ et la valeur certifiée $(2 \mu \mathrm{g} / \mathrm{kg})$. La méthode de dosage d'aflatoxine B1 dans les pâtes d'arachide par HPLC peut être considérée comme acceptable pour une analyse de routine.

(C) 2018 International Formulae Group. All rights reserved.

Mots clés: Aflatoxine B1, pâte d'arachide, méthode de validation.

\section{Validation of the method for determining Aflatoxin B1 in groundnut pastes sold in the markets of Abidjan (Côte d'Ivoire)}

\begin{abstract}
This study was carried out to validate the method of dosage of Aflatoxin B1 in the groundnut pastes for high performance liquid chromatography, according to the NFV03-110 Mai 2010 standard. The tests of the validation were performed out starting from ten samples. The linearity of calibration established between 0 and $3.0 \mu \mathrm{g} / \mathrm{L}$ was proven by determining the square of the coefficient of correlation of Pearson $\left(\mathrm{R}^{2}=0.9985\right)$. The limits of detection and quantification were respectively of $0.01 \mu \mathrm{g} / \mathrm{kg}$ and $0.02 \mu \mathrm{g} / \mathrm{kg}$. The coefficients of variation of the tests of repeatability and reproducibility are respectively $3.4 \%$ and $3.79 \%$, lower than $5 \%$. The rate of recovery was estimated at $107.6 \%$. The test of conformity did not show a significant difference $(\mathrm{P}>$
\end{abstract}


$0,05)$ between the average concentration in aflatoxine B1 $(2.02 \pm 1.44 \mu \mathrm{g} / \mathrm{kg})$ and that of the certified value $(2$ $\mu \mathrm{g} / \mathrm{kg}$ ). The method of quantifying aflatoxin B1 in peanut paste by using HPLC can be regarded as acceptable for an analysis of routine.

(C) 2018 International Formulae Group. All rights reserved.

Keywords: Aflatoxin B1, groundnut paste, validation method.

\section{INTRODUCTION}

L'arachide (Arachis hypogaea) est une plante oléagineuse appartenant au genre Arachis, de la famille des Fabaceae qui inclue la plupart des graines légumineuses et ayant une haute importance économique et nutritive (Eke-Ejiofor et al., 2012 ; Noba et al., 2014). C'est une plante tropicale originaire de l'Amérique du Sud qui a été introduite en Afrique, en Asie et en Europe (Ferguson et al., 2005). L'arachide est la cinquième culture parmi les oléagineux les plus importants dans le monde (Sanginga et Bergvinson, 2015), la deuxième source d'huile comestible (Knoden et al., 2003), la troisième source importante de protéines végétales et la douzième production végétale dans le monde (FAOSTAT, 2008).

En Afrique, la vente d'arachide est une activité génératrice de revenus pour les populations aussi bien en milieu rural qu'en urbain (FAO, 2007 ; Diedhiou et al., 2012). L'arachide est principalement transformée en huile, pâte, farine et divers dérivés qui entrent dans la composition de produits alimentaires. C'est une bonne source de lipides, de protéines, de minéraux, de carbohydrates, de fibres et de vitamine E (Griel et al., 2004).

Malgré ses potentialités économiques et nutritives, l'arachide rencontre d'énormes difficultés pour sa récolte et son stockage. Selon Chapeland-Leclerc et al. (2005), l'arachide est l'un des oléagineux le plus contaminé par les moisissures toxinogènes le plus souvent pendant la culture, la récolte et au cours du stockage. Par ailleurs, selon Wagacha et al. (2013), certaines moisissures comme les genres Aspergillus, Penicillium et Fusarium sont capables de produire des toxines dites mycotoxines dans les graines d'arachide et ses dérivés qui peuvent se révéler très toxiques pour le consommateur. Or, selon la FAO (2013), l'élimination complète des mycotoxines dans les produits alimentaires s'avère quasi-impossible en raison de leur stabilité thermique.

En Afrique subsaharienne et particulièrement en Côte d'Ivoire, la pâte d'arachide est produite essentiellement de façon artisanale et commercialisée directement sur les marchés sans aucun contrôle des pouvoirs publics. Or, des études menées dans certains pays notamment le Mali, le Bénin, le Nigéria et le Kenya ont révélé sa contamination par des mycotoxines (Adjou et al., 2012 ; Ndung'u et al., 2013). Parmi ces mycotoxines, l'aflatoxine B1 est celle qui est caractérisée par la toxicité la plus élevée et la plus cancérogène qui peut se trouver dans beaucoup de produits alimentaires, notamment l'arachide et ses dérivés (Soleimany et al., 2012). Le contrôle sanitaire impose que le niveau de contamination des pâtes d'arachide par l'aflatoxine B1 soit déterminé avec précision pour une prise de décision par les pouvoirs public. La première étape de cette démarche est donc la validation de la méthode de détermination d'aflatoxine B1 utilisant comme principe la chromatographie liquide haute performance.

\section{MATERIEL ET METHODES Matériel d'étude}

Le matériel d'étude est constitué de dix échantillons de pâte d'arachide collectée dans différents marchés de la ville d'Abidjan ayant pour laltitude : 5.3364 et longitude : -4.0266 avec $5^{\circ} 20^{\prime} 11^{\prime \prime}$ Nord et $4^{\circ} 1^{\prime} 36^{\prime \prime}$ Ouest. 


\section{Réactifs}

Les réactifs utilisés sont tous de grade analytique. Il s'agit de solutions étalon d'aflatoxine B1 notamment, méthanol grade HPLC (Fisher Bioblock Scientific, France), acétonitrile grade HPLC (Fisher Bioblock Scientific, France), acide acétique (Sigma Bioblock Scientific, France), phosphate de potassium dihydrogéné (Fulka, France), hydrogénocarbonate de sodium (Fulka, France).

\section{Appareillage}

Le dosage d'aflatoxine B1 a été réalisé sur un Chromatographe Liquide Haute Performance (Shimadzu, Kyoto, Japon) muni d'un détecteur de fluorescence, d'une pompe à vide, d'un injecteur automatique, d'une colonne Shim-pack CLCG-ODS C18 (4 $\mu \mathrm{m} \mathrm{x}$ $150 \mathrm{~mm} \quad \mathrm{x} \quad 4,6 \mathrm{~mm})$ et un logiciel d'exploitation (Kroma 3000). Les conditions opératoires sont présentées dans le Tableau 1.

\section{Méthode de validation}

La validation de la méthode d'analyse a été réalisée selon la méthode de l'Association Française de Normalisation (NF V03-110 Mai 2010). Cette procédure comprend l'étude de la linéarité de la droite d'étalonnage, la détermination de la limite de détection et la quantification, le calcul du coefficient de variation pour les essais de répétabilité et de reproductibilité, et le calcul du taux de récupération pour l'exactitude des essais.

\section{Test de la linéarité}

L'étude de la linéarité d'aflatoxine B1 a été testée entre 0 et $3,0 \mu \mathrm{g} / \mathrm{L}$ en utilisant 5 points d'étalonnage $(0 \mu \mathrm{g} / \mathrm{L}, 0,2 \mu \mathrm{g} / \mathrm{L}, 0,6$ $\mu \mathrm{g} / \mathrm{L}, 1,3 \mu \mathrm{g} / \mathrm{L}$ et $3,0 \mu \mathrm{g} / \mathrm{L}$ ). Cinq points distincts ont été effectués.

\section{Limite de détection et limite de quantification}

Les limites de détection (LD) et de quantification (LQ) ont été calculées à partir du blanc matrice et 10 essais distincts ont été analysés par CLHP.

$\mathrm{LD}=$ Moyenne du blanc +3 fois écart type $(\sigma)$.

$\mathrm{LQ}=$ Moyenne du blanc +10 fois écart type $(\sigma)$.

\section{Test de répétabilité et de reproductibilité}

Pour tester la répétabilité, les extraits d'un même échantillon de concentration à 0,5 $\mu \mathrm{g} / \mathrm{L}$ ont été injectés dix fois le même jour dans le système chromatographique. Le coefficient de variation (CV) est obtenu en faisant l'écart type des résultats de concentrations sur la moyenne des mêmes concentrations calculées.

Pour la reproductibilité, un échantillon de concentration $5 \mu \mathrm{g} / \mathrm{L}$ est injecté chaque jour dans le système chromatographique pendant dix jours (soit 10 injections). Le coefficient de variation $(\mathrm{CV})$ est calculé en faisant l'écart type des résultats de concentrations sur la moyenne des mêmes concentrations calculées.

\section{Taux de récupération ou de recouvrement \\ Dix prises d'essais distinctes} d'échantillons de référence de pâte d'arachide ont été analysées pour apprécier le taux de récupération par la méthode de détermination d'aflatoxine B1. Après extraction, purification et injection au système chromatographique, les concentrations calculées ont permis de déterminer le taux de récupération en faisant la concentration lue de la matrice injectée sur la concentration théorique.

\section{Méthode de dosage d'aflatoxine B1}

L'aflatoxine $\mathrm{B} 1$ a été déterminée selon les méthodes AOAC (2005) comprenant les étapes d'extraction, de purification sur colonne d'immuno-affinité et de quantification à l'aide d'un étalonnage après analyse par Chromatographie Liquide Haute Performance. 
Une quantité de $25 \mathrm{~g}$ de pâte d'arachide a été additionnée à $100 \mathrm{~mL}$ d'une solution de méthanol/eau $(80: 20, \mathrm{v} / \mathrm{v})$ dans un pot à centrifuger. Le mélange a été homogénéisé à l'aide d'un agitateur (Ultra Turax, France) pendant 3 minutes. L'homogénéisât a été centrifugé à 4000 tours/minutes pendant 5 minutes puis filtré sur papier Whatman $\mathrm{N}^{\circ} 4$ dans des Erlenmeyers de $25 \mathrm{~mL}$. Après filtration, un volume de $3 \mathrm{~mL}$ du filtrat a été additionné à $32 \mathrm{~mL}$ d'une solution tampon phosphate saline (PBS); soit un volume total d'extraction de $35 \mathrm{~mL} .30 \mathrm{~mL}$ d'extrait ont été déposés sur une colonne d'immunoaffinité (Vicam, USA) à un débit de $1,5 \mathrm{~mL} / \mathrm{min}$. La colonne a été ensuite lavée avec $10 \mathrm{~mL}$ de PBS à un débit de $2 \mathrm{~mL} / \mathrm{min}$; l'air dans la colonne a été éliminé grâce à une pompe à vide pendant 30 secondes. Les aflatoxines ont été éluées avec $1,5 \mathrm{~mL}$ d'une solution d'acétonitrile en trois étapes de 0,5 $\mathrm{mL}$ séparées d'une pause d'une minute entre chaque étape, à un débit de $0,5 \mathrm{~mL} / \mathrm{min}$. L'éluât a été dilué avec $1,5 \mathrm{~mL}$ de la solution
PBS sur la colonne à un débit de $0,5 \mathrm{~mL} / \mathrm{min}$. L'éluât a été ensuite recueilli dans un tube de $2 \mathrm{~mL}$ et séché sous azote à $40{ }^{\circ} \mathrm{C}$. Le résidu obtenu a été dissout dans $500 \mu \mathrm{L}$ de méthanol, puis le dosage a été effectué par Chromatographie Liquide Haute Performance.

\section{Analyse statistique}

Les différents résultats obtenus ont été traité avec le logiciel Statistica version 7.1. En cas de différence significative entre les paramètres étudiés, une analyse de variance (ANOVA) a été effectuée et le classement des moyennes a été fait selon le test de Duncan au seuil de signification $\alpha$ de 5\%. Les différences sont considérées comme significatives pour des valeurs de $\mathrm{P}<0,05$. Les données ont été transmises sur Excel et les résultats ont été présentés sous forme de graphique et de tableaux.

Tableau 1: Conditions chromatographiques.

\begin{tabular}{ll}
\hline Chromatographe & Conditions opératoires \\
\hline Colonne & Shim-pack CLCG-ODS C18, $4 \mu \mathrm{m}, 150 \mathrm{~mm}$ x 4,6 mm \\
Phase mobile & Méthanol/acétonitrile $(50: 50 ; \mathrm{v} / \mathrm{v})$ \\
Débit & $0,5 \mathrm{~mL} / \mathrm{min}$ \\
Température & $40^{\circ} \mathrm{C}$ \\
Volume injecté & $20 \mu \mathrm{l}$ \\
Détecteur & Fluorescence : $\lambda$ excitation : $365 \mathrm{~nm} ; \lambda$ émission $435 \mathrm{~nm}$ \\
Durée d'analyse & 15 minutes \\
\hline
\end{tabular}




\section{RESULTATS}

Les résultats de la validation sont consignés dans le Tableau 2. Le carré du coefficient de corrélation de Pearson $\left(\mathrm{R}^{2}\right)$ calculé pour apprécier la linéarité est de 0,9985. Le test de linéarité a montré une distribution normale sur toute la gamme d'étalonnage de 0 à $3,0 \mu \mathrm{g} / \mathrm{L}$. Les limites de détection et de quantification respectivement $0,01 \mu \mathrm{g} / \mathrm{kg}$ et $0,02 \mu \mathrm{g} / \mathrm{kg}$ ont montré une sensibilité élevée de la technique utilisée pour la détermination. Les coefficients de variation calculés ont été de 3,4\% pour le test de répétabilité et de $3,79 \%$ pour le test de reproductibilité. Le taux de récupération obtenu a été de 107,6\%. Le test de conformité n'a pas montré de différence significative au risque 0,05 entre la concentration moyenne obtenue dans les échantillons de référence de pâte d'arachide $(2,02 \pm 1,44 \mu \mathrm{g} / \mathrm{kg})$ et la valeur certifiée $(2$ $\mu \mathrm{g} / \mathrm{kg})$.

La Figure 1 présente le chromatogramme d'un échantillon de référence contenant l'aflatoxine $\mathrm{B} 1$ à une concentration de $5 \mu \mathrm{g} / \mathrm{L}$ et la Figure 2, la courbe d'étalonnage.

Tableau 2: Validation de la méthode de détermination d'aflatoxine B1.

\begin{tabular}{ll}
\hline Paramètres & Résultats \\
\hline Linéarité (coefficient de corrélation, $\mathrm{R}^{2}$ ) & 0,9985 \\
Limite de détection (LOD) & $0,01 \mu \mathrm{g} / \mathrm{kg}$ \\
Limite de quantification (LOQ) & $0,02 \mu \mathrm{g} / \mathrm{kg}$ \\
Répétabilité (Coefficient de variation, CV) & $3,4 \%$ \\
Reproductibilité (Coefficient de variation, CV) & $3,79 \%$ \\
Taux de récupération & $107,6 \%$ \\
Concentration d'aflatoxine B1 obtenue dans les échantillons de référence & $2,02 \pm 1,44^{\mathrm{a}} \mu \mathrm{g} / \mathrm{kg}$ \\
Valeur certifiée d'aflatoxine B1 & $2^{\mathrm{a}} \mu \mathrm{g} / \mathrm{kg}$ \\
\hline
\end{tabular}

Les valeurs portant une même lettre sur une même colonne ne présentent pas une différence significative $(\mathrm{P}<5 \%$ ) selon le test de Duncan.

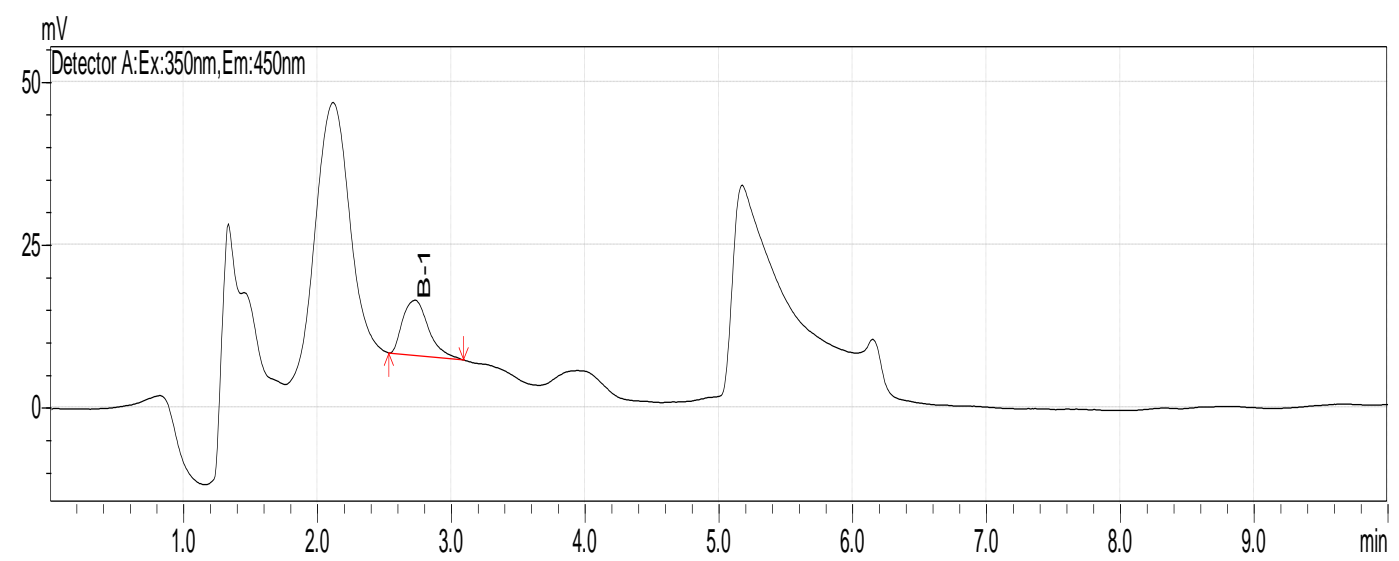

Figure 1: Chromatogramme d'un échantillon de référence contaminée à $5 \mu \mathrm{g} / \mathrm{L}$ d'aflatoxine B1 . 


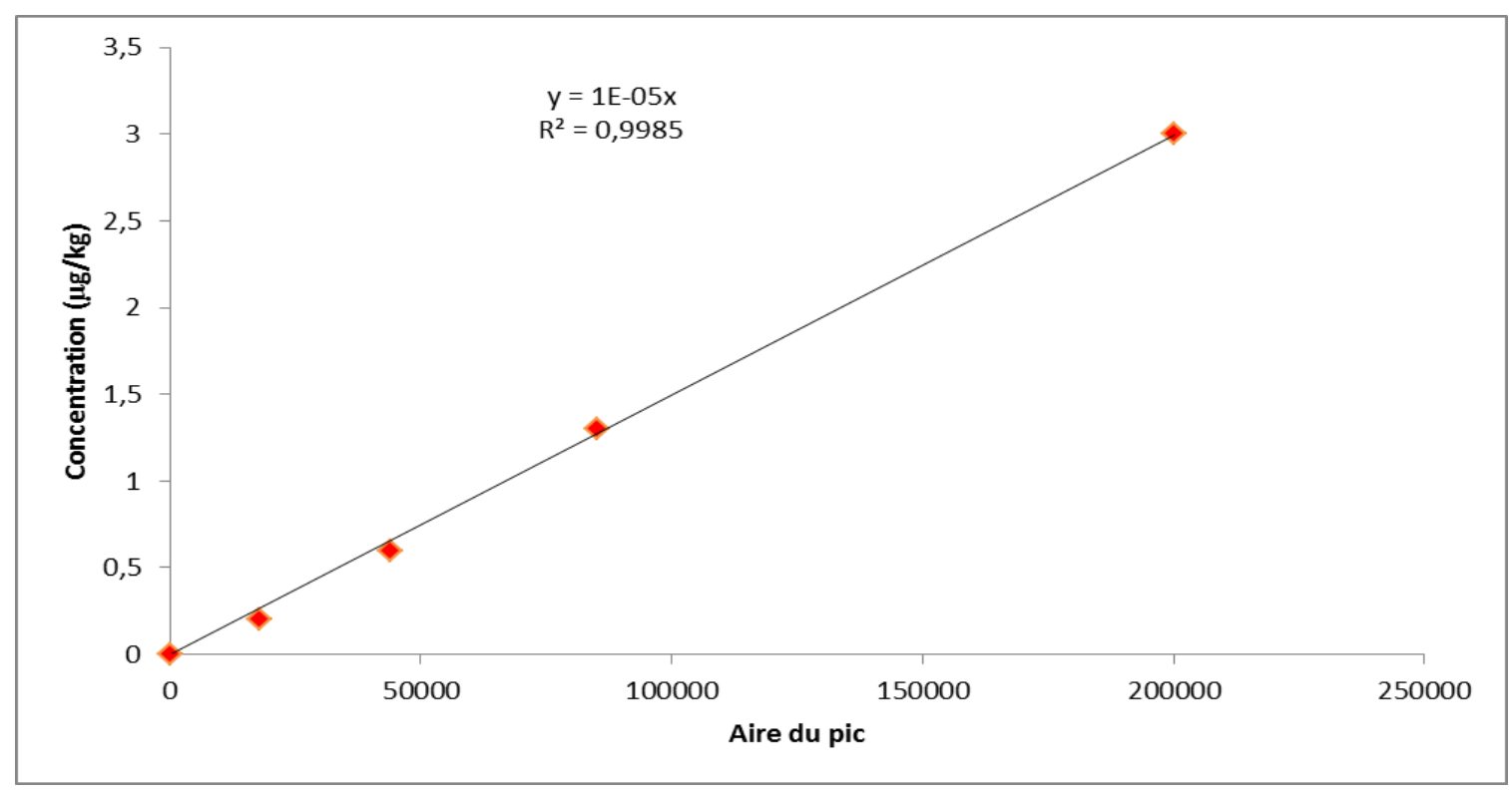

Figure 2: Courbe d'étalonnage d'aflatoxine B1.

\section{DISCUSSION}

La méthode utilisée pour cette étude a été validée en termes de linéarité, de limite de détection et de limite de quantification, de précision (tests de répétabilité et de reproductibilité) et du taux de récupération (rendement d'extraction). Ces différents paramètres de validation sont couramment utilisés pour la validation de la méthode de détermination des aflatoxines par chromatographie liquide haute performance (Alvarado-Hernández et al., 2016; Gurav et Medhe, 2018), mais aussi recommandés par ICH (International Conferences Harmonisation) et FDA (Food and drug administration) (Adhikari et Singh, 2016). Le test de la linéarité met en évidence la distribution normale sur toute la gamme d'étalonnage entre de 0 à $3,0 \quad \mu \mathrm{g} / \mathrm{L}$ d'aflatoxine B1. Le coefficient de corrélation obtenu pour la droite de régression est supérieur à 0,990 , de cette manière on peut conclure que la méthode utilisée est linéaire dans les domaines de concentrations étudiées comme rapportée par Dhib (2011). Les résultats obtenus pour les limites de détection et de quantification s'avèrent satisfaisants. La méthode est donc applicable et permet de détecter cette substance avec une sensibilité testée satisfaisante. Ces résultats corroborent ceux de Yazdanpanah et al. (2013) qui ont obtenu les limites de détection et de quantification respectives de $0,01 \mu \mathrm{g} / \mathrm{kg}$ et de $0,03 \mu \mathrm{g} / \mathrm{kg}$ pour la validation de la méthode de détermination d'aflatoxine B1 dans l'arachide. Les coefficients de variation calculés pour la matrice des échantillons des tests de répétabilité et de reproductibilité sont inférieurs à $5 \%$, donc acceptables. On peut dire que la méthode est donc applicable à la substance analysée. Le taux de récupération obtenu dans cette étude se situe dans la gamme recommandée (70-110\%) à l'échelle internationale, la méthode est donc applicable pour ce domaine de concentration. Les résultats obtenus sont comparables à ceux d'autres auteurs Baltac et al. (2013) et Yazdanpanah et al. (2013) qui ont obtenu respectivement 80 à $105 \%$ et 98,1 à $102,6 \%$ du taux de récupération pour la validation de la méthode de détermination d'aflatoxine B1 dans l'arachide. Les ajouts dosés et la détermination des concentrations certifiées des échantillons de référence ont aussi montré l'exactitude des résultats du dosage des différents échantillons car aucune différence 
significative n'a été observée avec le test de conformité. Par conséquent, les résultats des tests de validation sont conformes aux valeurs indiquant l'acceptabilité d'une technique d'analyse comme indiquée par les experts du comité mixte FAO/AIEA (1997) et documentées dans la collection des méthodes normalisées de l'Association Américaine de Santé Publique (APHA, 2005).

\section{Conclusion}

L'étude réalisée a présenté les résultats de l'application de la méthode de chromatographie liquide haute performance pour l'analyse d'aflatoxine B1. La procédure de validation a confirmé que la méthode proposée pour la détermination d'aflatoxine B1 dans les pâtes d'arachide a donné des résultats satisfaisants avec des valeurs acceptables pour une technique d'analyse à l'échelle internationale. La validation est indispensable à l'évaluation du risque des mycotoxines dans les denrées alimentaires et particulièrement dans les pâtes d'arachide vendues dans les marchés afin de prévenir et de veiller à la sécurité sanitaire des aliments pour le consommateur ivoirien et de la qualité du produit mis à la disposition dans les marchés locaux.

\section{CONFLIT D'INTERETS}

Les auteurs déclarent qu'il n'y a aucun conflit d'intérêts.

\section{CONTRIBUTIONS DES AUTEURS}

ZBIAB a assuré la collecte, les manipulations expérimentales, l'analyse des données et la rédaction du manuscrit. FC, DMK, MK et RKN ont participé à l'analyse des données et la correction de l'article. Tous les auteurs ont lu et approuvé le manuscrit définitif.

\section{REMERCIEMENTS}

Les auteurs remercient tous ceux qui ont participé à ce travail (étudiants, vendeuses de pâte d'arachide, techniciens, etc.).

\section{REFERENCES}

Adhikari R, Singh BH. 2016. A Review on Method Development and Validation using HPLC. International Journal for Pharmaceutical Research Scholars, 5(3): 167-172.

Adjou SE, Yehouenou B, Sossou CM, Soumanou MM, Souza CA. 2012. Occurrence of mycotoxins and associated mycoflora in peanut cake product (kulikuli) marketed in Benin. African Journal of Biotechnology, 11(78): 14354-14360. DOI: http://dx.doi.org/10.5897/AJB12.324

AFNOR (Association Française de Normalisation), 2010. Analyse des produits agricoles et alimentaires protocole de caractérisation en vue de la validation d'une méthode d'une méthode d'analyse quantitative par construction du profil d'exactitude. NF V03-110 Mai 2010. (Indice de classement : V03-110). Paris : Edition AFNOR. p. 57.

Alvarado-Hernández JR, Carvajal-Moreno M, Rojo-Callejas F, Ruiz-Velasco S. 2016. Aflatoxins in Natural Peanuts (Arachis hypogaea L.) of Mexico: Validation of the Biochemical Methods for Extraction and Quantification. Journal of Plant Biochemistry Physiology, 4(2): 1-11. DOI: 10.4172/2329-9029.1000168

AOAC (Association of Official Analytical Chemists) 2005. Aflatoxin M1 in liquid milk, immunoaffinity column by liquid chromatography. Official Methods of Analysis of AOAC International (18th edition). AOAC: USA; 45-47.

APHA (American Public Health Association) 2005. Standard methods for the examination of water and wastewater. American Public Health Association. Franson M. A. H., $27^{\text {st }}$ edition, Washington DC, p. 72.

Baltac C, İlyasoğlu H, Yüksel F. 2013. SingleLaboratory Validation for the Determination of Aflatoxin $\mathrm{B}_{1}, \mathrm{~B}_{2}, \mathrm{G}_{1}$, and $\mathrm{G}_{2}$ in Foods Based on Immunoaffinity Column and Liquid Chromatography with Postcolumn Derivatization and Fluorescence Detection. Food Analytical Methods, 6(1): 36-44.

Chapeland-Leclerc F, Papon N, Noël T, Villard J. 2005. Moisissures et risques 
alimentaires (mycotoxicoses). Revue Française des Laboratoires, p. 373

Dhib A. 2011. Validation d'une Méthode d'Analyse des pesticides dans les tomates fraîches par Chromatographie en Phase Gazeuse associée à un comptage par Scintillation Liquide. Diplôme d'Ingénieur, Université de Tunis, Tunis, p. 59.

Diedhiou PM, Bandyopadhyay R, Atehnkeng J, Ojiambo PS. 2011. Aspergillus colonization and aflatoxin contamination of maize and sesame kernels in two agroecological zones in Senegal. Journal of Phytopathology, 159(4): 268-275. DOI: http://dx.doi.org/10.1111/j.14390434.2010.01761.x

Eke-Ejiofor J, Kiin-Kabari DB, Chukwu EC. 2012. Effect of processing method on the proximate, mineral and fungi properties of groundnut (Arachis hypogea). Seed Journal of Agricultural and Biological Science, 3: 257-261.

FAO (Fond des Nations Unies pour l'Alimentation et de l'Agriculture) 2013. Programme mixte FAO/OMS sur les normes alimentaires. Comité du codex sur les contaminants dans les aliments, septième session, Moscou (Russie) p. 19

FAO (Fond des Nations Unies pour l'Alimentation et de l'Agriculture) 2007. Etude sur la commercialisation des produits maraichers et céréaliers et identification de nouveaux débouchés dans la région des savanes. p. 95

FAOSTAT (Food and Agriculture Organization of the United Nations) 2008. Statistics Food. Production data. Available online at: http://faostat.fao.org.

FAO (Food and Agriculture Organization of the United Nations) 1997. Validation of analytical methods for food control. Report of a Joint FAO/AIEA Expert Consultation, FAO Food and Nutrition Paper $\mathrm{N}^{\circ}$ 68, FAO, Rome, Italie. p. 17

Ferguson ME, Jarvis A, Stalker HT, Williams DE, Guarino L, Valls JF, Pittman RN, Bramel PJ. 2005. Biogeography of wild Arachis (Leguminosae): distribution and environmental characterisation. Biodiversity and Conservation ,14: 17771798.

Griel AE, Eissenstat B, Juturu V, Hsieh G, Kris-Etherton PM. 2004. Improved diet quality with peanut consumption. Journal of the American College of Nutrition, 23: 660-668.

Gurav NP, Medhe S. 2018. Analysis of aflatoxins B1, B2, G1 and G2 in Peanuts: Validation Study. Analycal Chemistry, 17(2): 1-9.

Knoden JL, Dufour L, Bindelle J. 2003. Fabrication de Beurre de Cacahuète. Collection Manuels et Techniques : Belgique ; 14.

Ndung'u JW, Makokha AO, Onyango CA, Mutegi CK, Wagacha JM, Christie ME, Wanjoya AK. 2013. Prevalence and potential for aflatoxin contamination in groundnuts and peanut butter from farmers and traders in Nairobi and Nyanza provinces of Kenya. Journal of Applied Biosciences, 65: 4922-4934.

Noba K, Ngom A, Guèye M, Bassène C, Kane M, Diop I, Ndoye F, Mbaye MS, Kane A, Ba AT. 2014. L'arachide au Sénégal: état des lieux, contraintes et perspectives pour la relance de la filière. Oilseeds Crpos and Lipids Journal Organisation, 21(2): 1-5. DOI: $10.1051 /$ ocl/2013039

Sanginga N, Bergvinson D. 2015. Un plan d'action pour la transformation de l'agriculture africaine. Oléagineux et Niébé. p. 27.

Soleimany F, Jinap S, Abas F. 2012. Determination of mycotoxins in cereals by liquid chromatography tandem mass spectrometry. Food Chemistry, 130: 1055-1060.

Wagacha JM, Mutegi CK, Christie ME, Karanja LW, Kimani J. 2013. Changes in Fungal Population and Aflatoxin Levels and Assessment of Major Aflatoxin Types in Stored Peanuts (Arachis hypogaea Linnaeus). Journal of Food Research, 2(5): 10-23.

Yazdanpanah H, Zarghi A, Shafaati AR, Foroutan SM, Aboul-Fathi F, Khoddam A, Nazari F, Shaki F. 2013. Analysis of Aflatoxin B1 in Iranian Foods Using HPLC and a Monolithic Column and Estimation of its Dietary Intake. Iranian Journal of Pharmaceutical Research, 12: 83-89. 